\title{
Alterations in the Structure, Physicochemical Properties, and pH of Hepatocyte Lysosomes in Experimental Iron Overload
}

\author{
Brent M. Myers, Franklyn G. Prendergast, Ralph Holman, Susan M. Kuntz, and Nicholas F. LaRusso \\ Center for Basic Research in Digestive Diseases, Division of Gastroenterology and Internal Medicine, and Department of Biochemistry \\ and Molecular Biology, Mayo Clinic, Medical School and Foundation, Rochester, Minnesota 55905; \\ and the Hormel Institute, University of Minnesota, Austin, Minnesota 55912
}

\begin{abstract}
While hemochromatosis is characterized by sequestration of iron-protein complexes in hepatocyte lysosomes, little is known about the effects of excess iron on these organelles. Therefore, we studied the effects of experimental iron overload on hepatocyte lysosomal structure, physicochemical properties, and function in rats fed carbonyl iron. A sixfold increase ( $P$ $<0.0001)$ in hepatic iron and a fivefold increase in lysosomal iron $(P<0.01)$ was observed after iron loading; as a result, hepatocyte lysosomes became enlarged and misshapen. These lysosomes displayed increased $(P<0.0001)$ fragility; moreover, the fluidity of lysosomal membranes isolated from livers of iron-loaded rats was decreased $(P<0.0003)$ as measured by fluorescence polarization. Malondialdehyde, an end product of lipid peroxidation, was increased by $73 \%(P<0.008)$ in lysosomal membranes isolated from livers of iron-overloaded rats. While amounts of several individual fatty acids in isolated lysosomal membranes were altered after iron overload, cholesterol/ phospholipid ratios, lipid/protein ratios, double-bond index, and total saturated and unsaturated fatty acids remained unchanged. The $\mathrm{pH}$ of lysosomes in hepatocytes isolated from livers of iron-loaded rats and measured by digitized video microscopy was increased (control, 4.70 \pm 0.05 ; iron overload, $5.21 \pm 0.10 ; P<0.01$ ). Our results demonstrate that experimental iron overload causes marked alterations in hepatocyte lysosomal morphology, an increase in lysosomal membrane fragility, a decrease in lysosomal membrane fluidity, and an increase in intralysosomal pH. Iron-catalyzed lipid peroxidation is likely the mechanism of these structural, physicochemical, and functional disturbances. (J. Clin. Invest. 1991. 88:1207-1215.) Key words: hemochromatosis • lipid peroxidation • organelle damage
\end{abstract}

\section{Introduction}

Lysosomes are membrane bound, acidic organelles that contain hydrolytic enzymes capable of degrading virtually all cellu-

Some of this work has been published previously in abstract form (1987; Hepatology 7:1045; and 1988; Hepatology 8:1240) and was presented at the 1987 and 1988 meetings of the American Association for the Study of Liver Diseases, Chicago, IL.

Address correspondence to Nicholas F. LaRusso, M.D., Professor of Medicine, Associate Professor of Biochemistry and Molecular Biology, Center for Basic Research in Digestive Diseases, Mayo Clinic, 200 First Street S.W., Rochester, MN 55905.

Received for publication 22 February 1991 and in revised form 21 May 1991.

The Journal of Clinical Investigation, Inc.

Volume 88, October 1991, 1207-1215 lar constituents. In addition to intracellular digestion, lysosomes play a major role in transcellular transport, intracellular storage, and extracellular secretion (1). The integrity of lysosomal membranes is essential for optimal lysosomal function, including the fusion of lysosomes with other organelles and the prevention of release of degradative lysosomal enzymes into the cell cytoplasm (2). The fluidity of lysosomal membranes is also important for lysosomal-organelle fusion and for the movement of constituents across lysosomal membranes (3-6). Lysosomes normally maintain an internal $\mathrm{pH}$ of $\sim 4.7$ via a membrane bound, proton pump (7). The normal function and proper orientation of the proton pump in the lysosomal membrane are thought to be essential to maintaining the acidic intralysosomal pH necessary for the optimum activity of lysosomal hydrolases.

Hemochromatosis is a genetic disease characterized by the accumulation of iron within organs, including the liver (8). While a major feature of this disease is the selective sequestration of excess iron in hepatocyte lysosomes (9), the mechanism of sequestration and the consequences of excess iron on hepatocyte lysosomes is unknown. Untreated, hepatic iron overload causes fibrosis and cirrhosis by mechanisms which are currently obscure.

Previous work from our laboratory has shown that hepatocyte lysosomes play a major role in iron metabolism, particularly with regard to biliary iron excretion. We demonstrated that exocytosis of lysosomal contents, including iron, into biliary canaliculi is a major excretory pathway for excess hepatic iron after experimental iron overload (10). We also showed that experimental iron overload increased the fragility of hepatocyte lysosomes in proportion to the degree of hepatic iron overload. While the mechanism of this increased lysosomal membrane fragility was unclear from our work, others have suggested that the cytotoxic effects of metals, including iron, are mediated by metal-induced, membrane lipid peroxidation (11-13). Of particular note is the fact that the physiological consequences of the sequestration of excess iron in hepatocyte lysosomes has not been investigated. Therefore, to extend our previous observations, we hypothesized that the accumulation of excess iron in hepatocyte lysosomes detrimentally affects a variety of parameters of lysosomal integrity. To test this hypothesis, we systematically examined the effects of experimental iron overload on the morphology, physicochemical properties, and function of hepatocyte lysosomes using whole liver, isolated hepatocytes, isolated lysosomes, and isolated lysosomal membranes.

\section{Methods}

Experimental model of hepatic iron overload. Male Sprague Dawley rats ( $150 \mathrm{~g}$; Harlan Sprague Dawley, Inc., Indianapolis, IN) were used 
for all experiments and were fed either standard laboratory chow (Ralston Purina Co., St. Louis, MO) or chow (Teklad Premier Laboratory Diets, Madison, WI) containing $2 \%$ carbonyl iron (Ferronyl ${ }^{\mathrm{TM}}$; GAF Chemicals Corp., Wayne, NJ) for $6 \mathrm{wk}$ (11). After 12-16 h of fasting, either: (a) animals were killed by decapitation and livers removed immediately for subsequent analytical and preparative manipulation (see below); or $(b)$ hepatocytes were isolated by a standard collagenase perfusion method (see below). A total of 37 rats (control, $n=19$; iron loaded, $n=18$ ) were used for all experiments. To avoid possible storage effects, fresh tissue was immediately processed for all analytical procedures; in some circumstances (e.g., protein and lipid measurements), processed samples were stored at $-80^{\circ} \mathrm{C}$ for up to $6 \mathrm{wk}$ before assays were done.

Analytical procedures. Iron concentrations of whole liver and isolated lysosomes were measured by atomic absorption spectrophotometry (model 5000; Perkin-Elmer Corp., Norwalk, CT) as previously described (10). Protein measurements were performed with an assay using gamma globulin as a standard (Bio-Rad Laboratories, Richmond, CA) (14). The lysosomal enzyme, $N$-acetyl- $\beta$-glucosaminidase (B$\mathrm{Nag})^{1}$ (EC 2.3.1.30), was assayed spectrofluorometrically as previously described (15). Lysosomal fragility was assessed by measuring the latent activity of B-Nag in postnuclear supernates of rat liver as previously described in detail (16). Briefly, activity of B-Nag is measured in the presence (total activity) or absence (free activity) of a detergent (i.e., Triton X-100), and latency calculated as the ratio of total minus free activity to total activity expressed as a percentage. Under normal conditions, latency of B-Nag in rat liver is $\sim 80 \%$; under conditions where lysosomes are more fragile, latency will decrease.

After isolation of hepatic lysosomal membranes (see below), lysosomal membrane lipids were deproteinized by heating in isopropyl alcohol at $70^{\circ} \mathrm{C}$ and centrifuging at $500 \mathrm{~g}$ for $15 \mathrm{~min}$. Total lysosomal membrane cholesterol was measured using a commercial cholesterol oxidase assay as previously described (17). Total lysosomal membrane phospholipids were measured using a commercial phospholipase D, choline oxidase, and peroxidase assay as previously described (18).

Fatty acid analysis of lysosomal membrane lipids was performed with capillary gas chromatography as previously described (19). Membranes were extracted with chloroform:methanol (2:1) and phospholipids were separated by thin layer chromatography using petroleum ether:diethyl ether:acetic acid (80:20:1). The phospholipid band was transesterified with boron trifluoride methanol $(14 \% \mathrm{wt} / \mathrm{vol})$ at $85^{\circ} \mathrm{C}$ for $90 \mathrm{~min}$. Fatty acid methyl esters were extracted with petroleum ether and the percentage of individual fatty acids was determined with capillary gas chromatography using a bonded silica column (FFAP 2; Quadrex Corp., New Haven, CT). The double bond index was calculated by summing the products of mole fraction by number of double bonds per molecule for each fatty acid present in the sample (20).

Malondialdehyde, an end product of lipid peroxidation, was measured by the thiobarbituric assay (21). $250 \mu$ l of isolated lysosomal membrane was added to $500 \mu \mathrm{l}$ of $10 \%$ TCA in $0.25 \mathrm{~N} \mathrm{HCl}$ and centrifuged at $500 \mathrm{~g}$ for $20 \mathrm{~min}$. $500 \mu \mathrm{l}$ of supernatant was added to $5 \mu \mathrm{l}$ of $2 \%$ butylated hydroxytoluene and $1.0 \mathrm{ml}$ of $0.67 \%$ thiobarbituric acid, heated to $100^{\circ} \mathrm{C}$ for $15 \mathrm{~min}$, and absorbance measured at $532 \mathrm{~nm}$ with a spectrophotometer. Malondialdehyde bis(dimethyl acetal) was used as a standard.

Transmission electron microscopy and acid phosphatase cytochemistry. Transmission electron microscopy of control and iron-loaded livers was performed as previously described (10). For experiments with isolated lysosomes, isolated lysosomes were fixed for $1 \mathrm{~h}$ in cold $2.5 \%$ glutaraldehyde in $0.1 \mathrm{M}$ phosphate buffer, $\mathrm{pH} 7.2$, rinsed in buffer, postfixed in cold $1 \% \mathrm{OsO}_{4}$ in $0.1 \mathrm{M}$ phosphate buffer, $\mathrm{pH} 7.2$, for $2 \mathrm{~h}$, and then rinsed twice in buffer. After postfixation, the specimens were rinsed once with $1 \%$ agar in $0.9 \%$ saline, centrifuged for $10 \mathrm{~s}$ at $8,000 \mathrm{~g}$, resuspended in fresh agar, and centrifuged again. Microfuge

1. Abbreviations used in this paper: B-Nag, $N$-acetyl- $\beta$-glucosaminidase; DPH, 1,6-diphenyl-1,3,5-hexatriene; FITC-Dex, FITC dextran; SIE, sucrose-imidazole-ethanol. tubes were placed on ice until the agar hardened. The tips of the microfuge tubes were cut off and the lysosomal pellets embedded in agar were removed. Liver sections, cubes, and lysosome pellets were dehydrated in a graded series of alcohols and embedded in Spurr (22). Acid phosphatase histochemistry was performed according to Gomori (23). Sections were examined with a transmission electron microscope (201; Philips Electronic Instruments, Inc., Mahwah, NJ). Quantitative morphometry with the IBAS 2000 system (Carl Zeiss Inc., Thornwood, NY) was used to measure the size of lysosomes in situ by determining cross-sectional area.

Isolation of lysosomes and lysosomal membranes. Lysosomes were isolated by isopycnic density centrifugation according to a modification of the method of Yamada et al. (1, 24). Briefly, livers were weighed and minced before homogenization in iced $250 \mathrm{mM}$ sucrose- $3 \mathrm{mM}$ imidazole-0.1\% ethanol (SIE) at pH 7.4. A postnuclear supernatant (E fraction) containing cellular organelles and cytosol was prepared as previously described (15). The $\mathrm{E}$ fraction was then incubated with 1 $\mathrm{mM} \mathrm{CaCl}{ }_{2}$ for $5 \mathrm{~min}$ at $37^{\circ} \mathrm{C}$; incubation with $\mathrm{CaCl}_{2}$ causes mitochondria to swell and become less dense, a maneuver which permits nearly complete separation of lysosomes from mitochondria $(1,24)$. The $E$ fraction was then layered on a self-forming gradient of Percoll (Pharmacia LKB Biotechnology Inc., Piscataway, NJ) and centrifuged at $80,000 \mathrm{~g}$ for $15 \mathrm{~min}$ using a TI 50.2 rotor in an ultracentrifuge (LZ-65B; Beckman Instruments, Inc., Fullerton, CA). Percoll was removed by washing with SIE and $1 \mathrm{mM}$ EDTA and by centrifuging at $75,000 \mathrm{~g}$ for 90 min using an SW28 rotor. Two additional washes with SIE and 1 mM EDTA followed by centrifugation at $10,000 \mathrm{~g}$ for $30 \mathrm{~min}$ in an SW41 rotor were performed. A 2-ml aliquot of lysosomes was removed and the remaining lysosomes were disrupted by hypoosmotic shock in $5 \mathrm{mM}$ Tris buffer; lysosomal membranes were then separated from lysosomal contents by centrifugation at $80,000 \mathrm{~g}$ for $90 \mathrm{~min}$ with an SW41 rotor. The distribution over the Percoll gradient of $N$-acetyl- $\beta$ glucosaminidase, malate dehydrogenase (a marker enzyme for mitochondria), and iron was measured to validate that this method could be satisfactorily applied to iron-loaded livers.

Lysosomal membrane fluidity. Fluidity of lysosomal membranes was estimated by measuring steady-state anisotropy using fluorescence polarization (25). Approximately $100 \mu \mathrm{g}$ protein of isolated lysosomes was added to $2.0 \mathrm{ml}$ of SIE buffer. $5 \mu \mathrm{l}$ of $1 \mathrm{mM}$ 1,6-diphenyl-1,3,5-hexatriene (DPH) in tetrahydrofuran was added to the solution and allowed to equilibrate for $1 \mathrm{~h}$ with frequent vortexing to evaporate the tetrahydrofuran. Steady-state anisotropy was measured in a spectrofluorometer (4800; SLM Instruments, Inc., Urbana, IL) at $25^{\circ} \mathrm{C}$ with polarization filters parallel and perpendicular to the excitation beam. Excitation was at $362 \mathrm{~nm}$ and emission at $420 \mathrm{~nm}$. Fluorescent lifetimes were measured by the phase-modulation method of Spencer and Weber (26) using a subnanosecond spectrofluorometer. An argon ion laser (2025; Spectra-Physics, Inc., Mountain View, CA) was used for excitation at $360 \mathrm{~nm}$. An optical nodule from a 4800 spectrofluorometer (SLM Instruments, Inc.) coupled with a frequency synthesizer (Programmed Test Sources, Inc., Littleton, MA) was used to measure the phase delay and modulation difference of sample versus the reference lifetime standard dimethyl POPOP (p-bis[2-(5-phenyloxazolyl)]benzene) in ethanol $\left(T_{\mathrm{f}} \mathrm{POPOP}=1.35 \mathrm{~ns}\right)$ with calculations performed with an interfaced PC (IBM Instruments Inc., Danbury, CT).

Lysosomal $\mathrm{pH}$. Lysosomal $\mathrm{pH}$ was measured by applying a fluorescent technique originally described by Ohkuma (27) to freshly isolated hepatocytes using digitized video microscopy. FITC dextran (FITCDex), a lysosomotropic pH-sensitive fluorescent probe (FD-70; Sigma Chemical Co., St. Louis, MO), was introduced into rats by intraperitoneal injection $(20 \mathrm{mg} / 150 \mathrm{~g}$ body $\mathrm{wt}) 16 \mathrm{~h}$ before the experiment. Hepatocytes were then isolated by a modification of the method of Berry and Friend (28) previously described by us (29). Hepatocyte viability was $85-90 \%$ by trypan blue exclusion and purity was $99 \%$ by electron microscopy.

To relate lysosomal $\mathrm{pH}$ to fluorescence, a standard curve for lysosomal $\mathrm{pH}$ was prepared in intact hepatocytes. A series of phosphate-citrate buffers with $\mathrm{pH}$ ranging from 4.5 to 7.0 was prepared using 
$\mathrm{KH}_{2} \mathrm{PO}_{4}$ and citric acid, and adjusting osmolarity to $300 \mathrm{mOsm}$ using an osmometer. To inhibit the ATP-dependent proton pump of lysosomes (27), metabolic inhibitors ( $50 \mathrm{mM} \mathrm{Na}$ azide and $50 \mathrm{mM}$ 2-deoxyglucose) were added to the $\mathrm{pH}$ buffers to suppress ATP production by hepatocytes. Ionophores (10 $\mu \mathrm{M}$ nigericin and $10 \mu \mathrm{M}$ monensin) were also added to equilibrate lysosomal $\mathrm{pH}$ with the $\mathrm{pH}$ of the buffers (30). Freshly isolated hepatocytes were added to the $\mathrm{pH}$ buffers and allowed to equilibrate for $10 \mathrm{~min}$ before $\mathrm{pH}$ measurements. No signifcant change in viability assessed by trypan blue exclusion was noted after addition of these agents within the timeframe of the experiment.

Fluorescence $\mathrm{pH}$ was measured using a fluorescent microscope interfaced with an IBAS 2000 image analysis system (Carl Zeiss Inc.). Hepatocytes isolated from control or iron-loaded rats were placed on a microscope slide with excitation wavelength at $495 \mathrm{~nm}$ (pH sensitive) and emission wavelength at $520 \mathrm{~nm}$. The same field of hepatocytes was excited at $450 \mathrm{~nm}$ (pH insensitive) and the fluorescent images at both excitation wavelengths were stored on the IBAS system. Background fluorescence was subtracted with the IBAS system and the fluorescence of individual hepatocytes was converted to gray scale values. Nonviable hepatocytes were edited from the stored images. The $495 / 450 \mathrm{~nm}$ fluorescence ratios were calculated and the lysosomal $\mathrm{pH}$ of the sample read from the standard curve of $\mathrm{pH}$ versus $495 / 450 \mathrm{~nm}$ ratio.

Cell fractionation of FITC-dextran-labelled hepatocytes. Although the FITC-Dex was present in hepatocyte vesicles after intraperitoneal injection as assessed by fluorescence microscopy, we wished to confirm that these vesicles were lysosomes. To do this, a normal rat was injected intraperitoneally with FITC-Dex $(20 \mathrm{mg} / 150 \mathrm{~g}$ body wt) and killed $16 \mathrm{~h}$ later. The subcellular distribution of FITC-Dex was determined by differential centrifugation of rat liver as previously described (31). A nuclear or $\mathrm{N}$-fraction and a postnuclear supernatant or E-fraction were prepared by low-speed centrifugation. The E-fraction was further separated into four additional fractions enriched in mitochondria (heavy mitochondrial or M-fraction), lysosomes (light mitochondrial or Lfraction), endoplasmic reticulum (P-fraction), and cytosol (final supernatant or S-fraction). Each fraction was assayed for total protein; the lysosomal marker enzyme, $N$-acetyl- $\beta$-glucosaminidase; the mitochondrial marker enzyme, malate dehydrogenase (32); the endoplasmic reticulum marker enzyme, microsomal esterase (33); the plasma membrane marker enzyme, alkaline phosphodiesterase I (33); and the cytosolic marker enzyme, lactate dehydrogenase (34). Relative specific activity was plotted versus cumulative percentage of protein for each fraction as previously described (31).

Statistical methods. Results from the two groups of rats (control and iron overload) were compared using a two-sample independent $t$ test with the Clinfo computer system. Fatty acid analyses were compared using normalcy ratios $(19,20) . P$ values of 0.05 or less were considered statistically significant.

\section{Results}

Body weight and liver weight. There were no significant differences in total body weights [control $(n=8), 253 \pm 19 \mathrm{~g}$ vs. iron overload $(n=8), 241 \pm 17 \mathrm{~g}$ ] or in wet liver weight (control, $7.6 \pm 0.7 \mathrm{~g}$ vs. iron overload, $8.0 \pm 0.5 \mathrm{~g}$ ) after $6 \mathrm{wk}$ of carbonyl iron feeding. We previously reported that there were no major changes in serum bilirubin and aspartate aminotransferase levels after iron overload for this time period (10).

Hepatic iron content and in situ morphology. $6 \mathrm{wk}$ of iron loading resulted in a sixfold increase in total hepatic iron concentration (Table I). We confirmed previous work from our laboratory using transmission electron microscopy in situ that hepatocyte lysosomes in iron-overloaded rats are enlarged, abnormally shaped, and increased in number (data not shown). In work here, we extended these in situ morphologic findings cytochemically and quantitatively. Specifically, iron-filled organelles in hepatocytes were also positive for acid phosphatase
Table I. Effect of Iron Overload on the Iron Concentration, Size, Physicochemical Properties, and Intralysosomal pH of Hepatocyte Lysosomes*

\begin{tabular}{|c|c|c|c|}
\hline & Control & Iron overload & $P$ value \\
\hline $\begin{array}{l}\text { Iron concentration, whole } \\
\text { liver ( } \mu \mathrm{g} / \mathrm{g} \text { dry liver) }\end{array}$ & $\begin{array}{l}455 \\
( \pm 44)\end{array}$ & $\begin{array}{c}2641 \\
( \pm 295)\end{array}$ & 0.0001 \\
\hline Size of lysosomes $\left(\mu \mathrm{m}^{2}\right)$ & $\begin{array}{l}0.0698 \\
( \pm 0.0021)\end{array}$ & $\begin{array}{l}0.0986 \\
( \pm 0.0042)\end{array}$ & 0.0001 \\
\hline $\begin{array}{l}\text { Iron concentration, } \\
\text { isolated lysosomes, }(\mu \mathrm{g} / \mathrm{g} \\
\text { protein) }\end{array}$ & $\begin{array}{r}1211 \\
( \pm 92)\end{array}$ & $\begin{array}{l}5716 \\
( \pm 881)\end{array}$ & 0.01 \\
\hline Latency $(\%)$ & $\begin{array}{c}78 \\
( \pm 2)\end{array}$ & $\begin{array}{l}46 \\
( \pm 4)\end{array}$ & 0.001 \\
\hline $\begin{array}{l}\text { Steady-state anisotropy of } \\
\text { lysosomal membranes }\end{array}$ & $\begin{array}{l}0.185 \\
( \pm 0.005)\end{array}$ & $\begin{array}{l}0.211 \\
( \pm 0.004)\end{array}$ & 0.003 \\
\hline $\begin{array}{l}\text { Fluorescent lifetimes } \\
\text { (nanoseconds) }\end{array}$ & $\begin{array}{l}7.66 \\
( \pm 0.15)\end{array}$ & $\begin{array}{l}7.62 \\
( \pm 0.18)\end{array}$ & NS \\
\hline $\begin{array}{l}\text { Cholesterol/phospholipid } \\
\text { ratio of lysosomal } \\
\text { membranes }\end{array}$ & $\begin{array}{l}1.20 \\
( \pm 0.31)\end{array}$ & $\begin{array}{l}1.29 \\
( \pm 0.42)\end{array}$ & NS \\
\hline $\begin{array}{l}\text { Protein/lipid ratio (mg/ } \\
\text { mg) of lysosomal } \\
\text { membranes }\end{array}$ & $\begin{array}{l}96.8 \\
( \pm 30.7)\end{array}$ & $\begin{array}{l}60.5 \\
( \pm 6.9)\end{array}$ & NS \\
\hline $\begin{array}{l}\text { Double-bond index of } \\
\text { lysosomal membrane } \\
\text { phospholipid fatty acids }\end{array}$ & $\begin{array}{l}1.87 \\
( \pm 0.06)\end{array}$ & $\begin{array}{l}1.92 \\
( \pm 0.13)\end{array}$ & NS \\
\hline $\begin{array}{l}\text { Polyunsaturated fatty acids } \\
\text { (percentage of lysosomal } \\
\text { membrane phospholipid } \\
\text { fatty acids) }\end{array}$ & $\begin{array}{l}47.1 \\
( \pm 1.6)\end{array}$ & $\begin{array}{c}49.0 \\
( \pm 3.1)\end{array}$ & NS \\
\hline $\begin{array}{l}\text { Saturated fatty acids } \\
\text { (percentage of lysosomal } \\
\text { membrane phospholipid } \\
\text { fatty acids) }\end{array}$ & $\begin{array}{l}44.1 \\
( \pm 1.9)\end{array}$ & $\begin{array}{l}40.8 \\
( \pm 3.2)\end{array}$ & NS \\
\hline $\begin{array}{l}\text { Chain length of lysosomal } \\
\text { phospholipid fatty acids }\end{array}$ & $\begin{array}{c}17.609 \\
( \pm 0.019)\end{array}$ & $\begin{array}{c}17.556 \\
( \pm 0.048)\end{array}$ & 0.05 \\
\hline $\begin{array}{l}\text { Melting point of lysosomal } \\
\text { membrane phospholipid } \\
\text { fatty acids }\end{array}$ & $\begin{array}{c}13.61 \\
( \pm 0.72)\end{array}$ & $\begin{array}{l}8.17 \\
( \pm 1.25)\end{array}$ & 0.001 \\
\hline $\begin{array}{l}\text { Malondialdehyde (nmol/ } \\
\text { mg lipid) of lysosomal } \\
\text { membranes }\end{array}$ & $\begin{array}{l}93.7 \\
( \pm 12.2)\end{array}$ & $\begin{array}{c}162.5 \\
( \pm 18.4)\end{array}$ & 0.008 \\
\hline Intralysosomal pH & $\begin{array}{l}4.70 \\
( \pm 0.05)\end{array}$ & $\begin{array}{l}5.21 \\
( \pm 0.10)\end{array}$ & 0.01 \\
\hline
\end{tabular}

* Data represent mean $( \pm$ SEM) from five to six animals in each group for each measurement.

reaction product, establishing unequivocally their identity as lysosomes (data not shown). Moreover, quantitative morphometry showed a significant increase in the size of lysosomes after iron overload (Table I).

Isolation of hepatic lysosomes from iron-loaded rats. Fig. 1 shows transmission electron micrographs of hepatocyte lysosomes isolated from control and iron-loaded rats. The high degree of purity and lack of contamination with other organelles is apparent. Lysosomes from iron-loaded rats are enlarged and irregular in shape compared to lysosomes isolated from controls. Lysosomes isolated from livers of control and iron- 

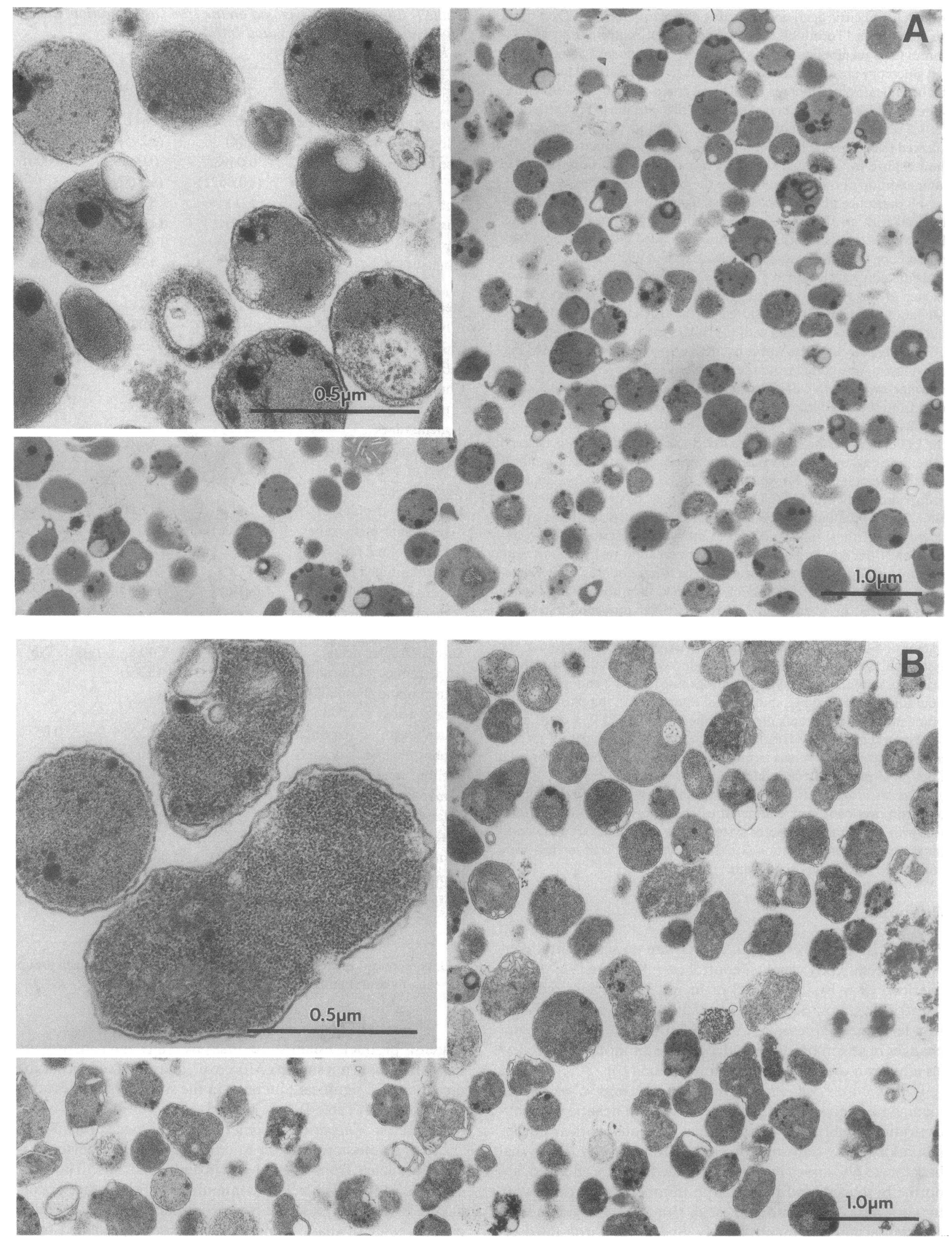

Figure 1. Lysosomes isolated from normal $(A)$ and iron-overloaded $(B)$ rat liver hepatocytes. $A$ and $B, \times 18,200 ;$ inset, $\times 61,880$. 
loaded rats stained positively for acid phosphatase (data not shown). This isolation procedure resulted in a $50 \%$ yield and a 45-fold enrichment in intact lysosomes as measured by the lysosomal marker enzyme, $N$-acetyl- $\beta$-glucosaminidase.

The distribution of $N$-acetyl- $\beta$-glucosaminidase, malate dehydrogenase, and iron on a gradient of Percoll is shown in Fig. 2. The mitochondrial marker enzyme is primarily in the upper (less dense) gradient region. The lysosomal enzyme and iron exhibit a bimodal distribution with the majority of both in the lower (more dense) gradient region; these fractions contain intact lysosomes (latency $>50 \%$ ) while the other (less dense) fractions in the upper gradient region represent free lysosomal enzyme (latency 0\%) and iron released during homogenization. The iron concentration of isolated hepatic lysosomes was increased fivefold $(P<0.01)$ (Table I).

Physiochemical properties of hepatocyte lysosomes. There was a decrease $(P<0.0001)$ in the latent activity of the lysosomal enzyme, $N$-acetyl- $\beta$-glucosaminidase, after iron loading reflecting an increase in lysosomal fragility (Table I).

Steady-state anisotropy of DPH in lysosomal membranes isolated from livers of iron-loaded rats increased $(P<0.003)$ (Table I). In contrast, no change was noted in fluorescent lifetimes of this probe after iron overload (Table I); this observation indicates that the observed increase in anisotropy in lysosomal membranes from livers of iron-loaded rats was due to a change in DPH membrane fluidity and not due to quenching of the fluorescent probe from excess iron. Adding ferritin to a postnuclear supernate ( $E$ fraction) of rat liver before the isolation procedure in amounts $(2,500 \mathrm{mg} / \mathrm{g}$ liver) approximating that seen in liver after $6 \mathrm{wk}$ of iron loading did not affect steady state anisotropy of isolated lysosomes (control, $0.190 \pm 0.005$; control and ferritin, $0.193 \pm 0.006$ ); this finding suggests that the observed change in anisotropy in lysosomal membranes isolated from livers of iron-loaded rats reflects iron-induced alterations in lysosomal membrane fluidity that occur in vivo rather than during the isolation procedure. In an attempt to explain the observed iron-induced alteration in lysosomal membrane fluidity, we examined several parameters affecting

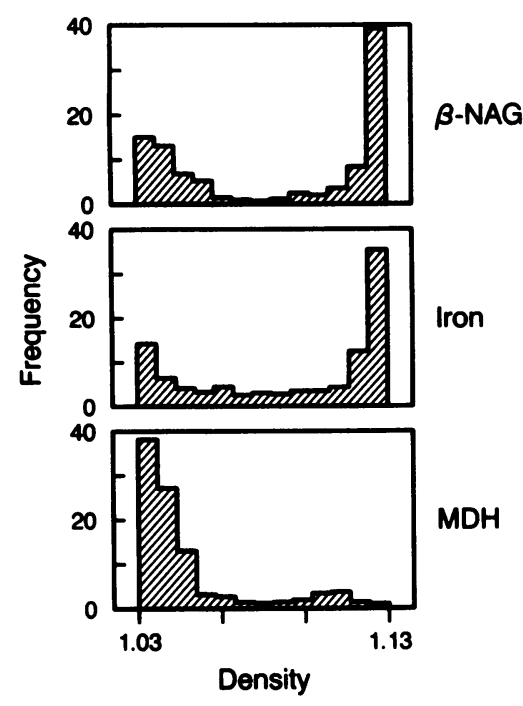

Figure 2. Distribution patterns of constituents after isopycnic centrifugation of $\mathrm{a} \mathrm{CaCl}_{2-}$ treated postnuclear supernatant of rat liver after iron loading. The average frequency of the components was calculated for each fraction as suggested by deDuve (31): $\mathrm{Q} / \mathrm{p} \Sigma \mathrm{Q}$, where $Q$ represents the activity found in the fraction, $\Sigma Q$ represents the total recovered activity, and $p$ represents the increment of density from the bottom to the top of the fraction. $\beta$ $N A G, N$-acetyl- $\beta$-gluco-

saminidase, a lysosomal enzyme; $M D H$, malate dehydrogenase, a mitochondrial enzyme; and iron, measured by atomic absorption spectrophotometry.

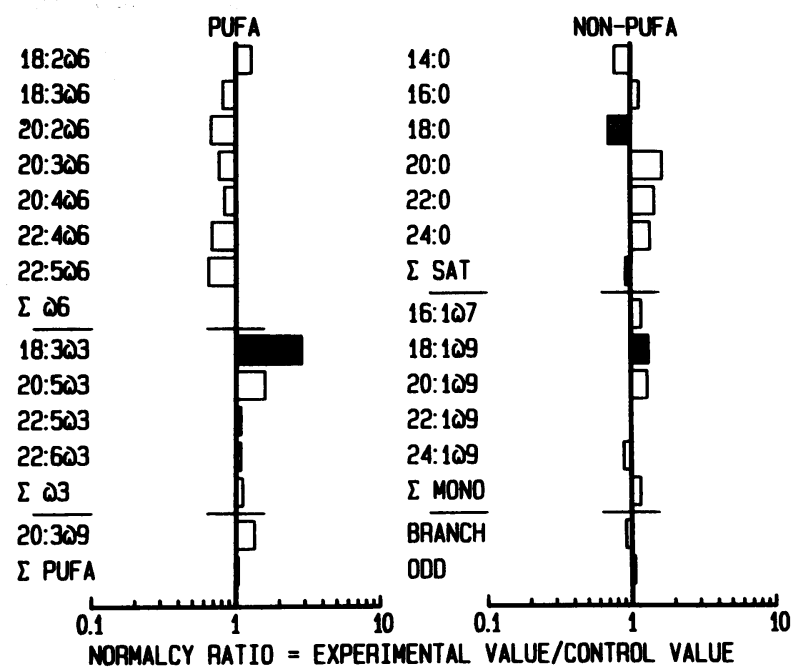

Figure 3. Polyunsaturated fatty acid (PUFA) and nonpolyunsaturated fatty acid (NON-PUFA) composition of lysosomal membranes after iron overload. Results are expressed as a normalcy ratio of iron overload/control. Shaded bars represent differences between overloaded animals and controls with significance at $P<0.01$. Open bars indicate nonsignificant differences.

membrane fluidity, including cholesterol/phospholipid ratio, protein/lipid ratio, and degree of unsaturation of fatty acids (Table I). There were no differences in the cholesterol/phospholipid ratio, the protein/lipid ratio, or in the double bond index of lysosomal phospholipid fatty acid, measures of the degree of unsaturation of fatty acids of isolated lysosomal membranes (Table I). Mean acyl chain length and mean melting point were significantly decreased $(P<0.05)$. Linolenic acid $(18: 3 \omega 3)$ was significantly elevated but $\omega 3$ acids derived from it were not significantly different from controls. Stearic acid (18:0) was

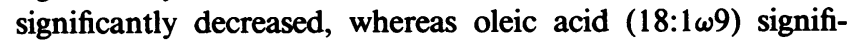
cantly increased (Fig. 3).

Malondialdehyde content of isolated lysosomal membranes increased $73 \%(P<0.008)$ after iron overload (Table I). The addition of ferritin to an $\mathrm{E}$ fraction from normal liver did not affect the malondialdehyde concentration $(84.7 \pm 10.1$ vs. $88.2 \pm 12.4 \mathrm{nmol} / \mathrm{mg}$ lipid) suggesting that the increase in malondialdehyde content of lysosomal membranes that occurred after iron loading is due to in vivo lipid peroxidation and not to iron-induced changes occurring during the isolation procedure.

Lysosomal pH. Fig. 4 shows fluorescent microscopy of hepatocytes isolated from the liver of a rat $16 \mathrm{~h}$ after the intraperitoneal injection of FITC-Dex. The FITC-Dex is sequestered in punctate, vesicular organelles. Tissue fractionation was performed to establish the nature of the organelles containing FITC-Dex. Fig. 5 shows the distribution of FITC-Dex and marker enzymes after fractionation of a rat liver by differential centrifugation $16 \mathrm{~h}$ after FITC-Dex injection. FITC-Dex displays a distribution pattern similar to the lysosomal enzyme, $N$-acetyl- $\beta$-glucosaminidase, and different from the marker enzymes for other organelles, providing biochemical evidence that the FITC-Dex is selectively sequestered in lysosomes. The distribution patterns of other marker enzymes are typical, attesting to the adequacy of the fractionation procedure. The $\mathrm{pH}$ of lysosomes in hepatocytes isolated from livers of iron-loaded 


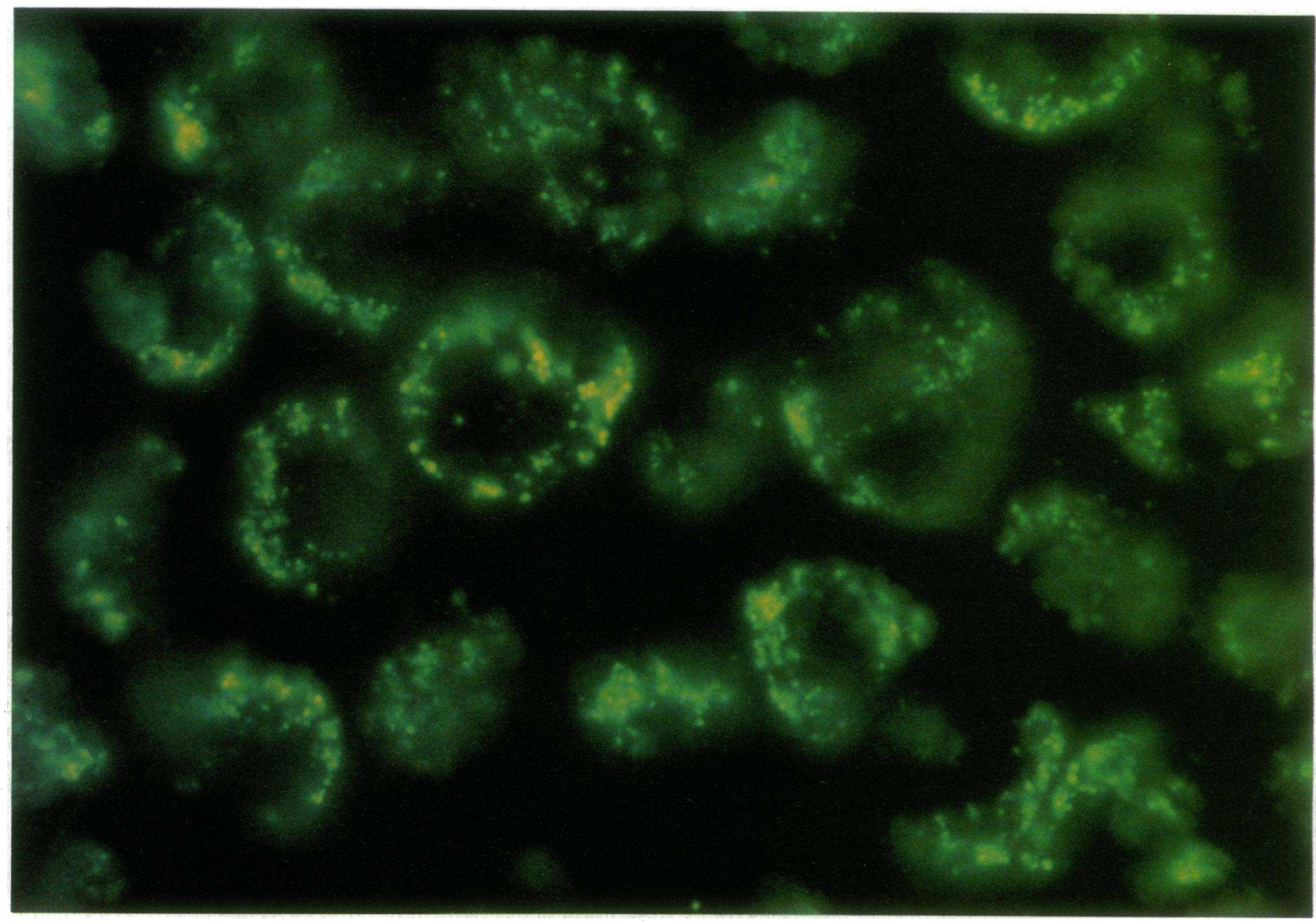

Figure 4. Fluorescence microscopy (excitation $495 \mathrm{~nm}$, emission $520 \mathrm{~nm}$ ) of hepatocytes isolated by collagenase perfusion $16 \mathrm{~h}$ after intraperitoneal injection of FITC-Dex $(20 \mathrm{mg} / 150 \mathrm{~g}$ body $\mathrm{wt})$. The FITC-Dex is sequestered within punctate vesicular organelles. $\times 630$.

rats was significantly increased (control, $4.70 \pm 0.05$; iron overload, 5.21 $\pm 0.10 ; P<0.01)$ as determined by digitized video microscopy (Table I).

\section{Discussion}

The major findings in this study relate to dramatic physicochemical alterations in hepatocyte lysosomes after experimental iron overload. We show that selective sequestration of iron occurs in hepatocyte lysosomes after iron loading and that the iron-loaded lysosomes are enlarged, misshapen, and fragile. Moreover, fluidity of the membranes of these abnormal lysosomes is decreased and their lipid composition is altered. The concomitant, iron-induced increase in malondialdehyde content of hepatic lysosomal membranes is consistent with the possibility that these physiochemical alterations and the associated functional disturbance of intralysosomal $\mathrm{pH}$ may be mediated by iron-catalyzed, membrane lipid peroxidation.

In situ, lysosomes in hepatocytes of iron-loaded rats were enlarged and irregular in shape as assessed by transmission electron microscopy. Moreover, iron-loaded lysosomes were filled with electron dense granules which others have identified as ferritin and hemosiderin (35). We have previously demonstrated that the number of lysosomes is increased in the pericanalicular region of the hepatocyte after iron loading (10). Our work here using quantitative morphometric techniques confirms and extends these in situ morphologic observations by demonstrating that these hepatocyte lysosomes are also increased in size. Moreover, we unequivocally established the identity of these iron-filled organelles as lysosomes by acid phosphatase cytochemistry. While no morphological evidence of liver damage was seen during our experimental period of iron loading, others have reported that iron loading greater than 1 yr will cause hepatic fibrosis (36). We have also shown previously that biliary iron excretion is increased after experimental iron overload and that this increase is closely coupled to the biliary excretion of lysosomal enzymes (10). These morphological and biochemical data, taken together, suggest that lysosomes are not only a storage organelle for excess iron but also serve a key role in its biliary excretion. Indeed, a recent report has demonstrated that biliary iron is increased in genetic hemochromatosis (37).

In addition to storing and excreting excess iron, the hepatocyte lysosome may also serve a role in protecting the cell from the potentially damaging effects of free metal ions, including iron. Ferric ions can induce peroxidation of membrane lipids by either reacting with physiological amounts of hydrogen peroxide to generate the highly reactive hydroxyl radical, or by complexing with oxygen producing perferryl and ferryl ions (38). These free radicals can also damage proteins and nucleic acids (39). Sequestered within lysosomes as iron ferritin, ferric ions may be prevented from initiating peroxidation of cytoplasmic lipids or lipids in other organelle membranes. There 


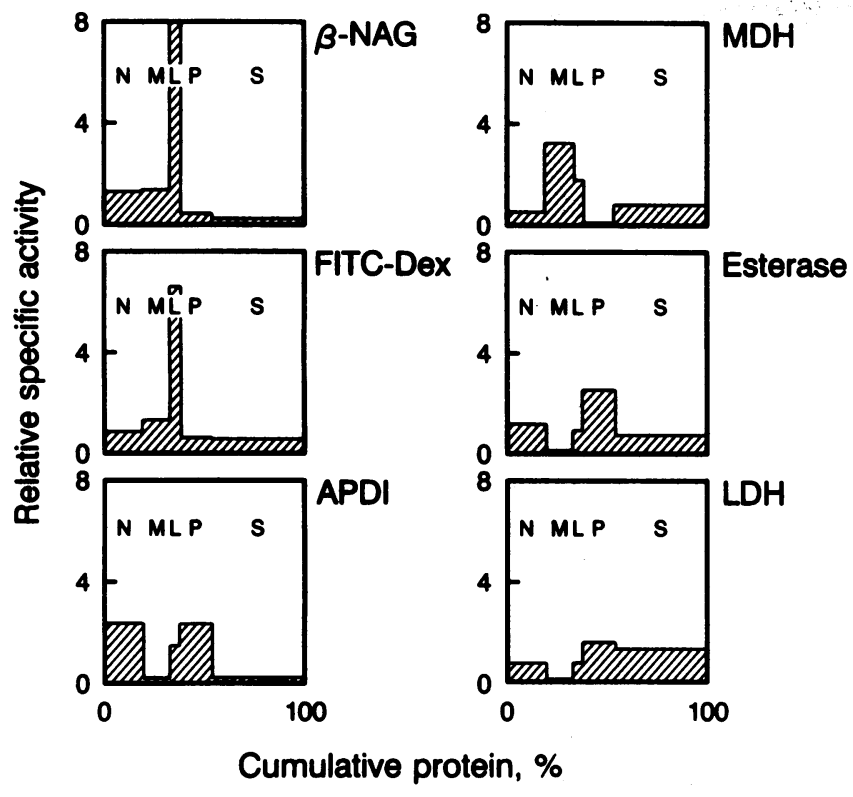

Figure 5. Distribution patterns of constituents after fractionation of a postnuclear supernatant of rat liver by differential centrifugation after injection of FITC-Dex. Fractions $(N, M, L, P, S)$ are plotted from left to right in order of the average coefficient of sedimentation of their subcellular components. Each fraction is represented separately on the ordinate by the relative specific activity of the constituent (percentage of total activity recovered/percentage of total protein recovered) and cumulatively on the abscissa by its percentage protein. $\beta$ - $N A G, N$-acetyl- $\beta$-glucosaminidase, a lysosomal enzyme; FITC-Dex, fluoroscein isothiocyanate dextran, a lysosomal pH probe; $M D H$, malate dehydrogenase, a mitochondrial enzyme; esterase, a microsomal enzyme; $A P D I$, alkaline phosphodiesterase I, a plasma membrane enzyme; and $L D H$, lactate dehydrogenase, a cytosolic enzyme. Recoveries of all constituents ranged from 85 to $121 \%$. N, nuclear; M, "heavy" mitochondrial; L, "light" mitochondrial, (lysosomal); P, microsomal; S, supernate.

appears to be a limit to the degree of this purported cytoprotection from iron that lysosomes can provide, however. Bacon has demonstrated that experimental iron overload causes mitochondrial injury as evidenced by increased lipid peroxidation and decreased ADP-stimulated mitochondrial respiration (40). The ability of lysosomes to protect the cell from iron-induced injury may be exceeded in two ways: first, the capacity of the lysosome to sequester iron and/or to excrete iron into bile may be exceeded; alternatively, the lysosomal membrane itself may be damaged by iron allowing leakage of iron into the cytoplasm. There is indirect evidence to support the second hypothesis; specifically, metal overloaded lysosomes from livers of patients with hemochromatosis are very fragile $(41,42)$. Furthermore, Peters has provided morphological evidence that this increase in hepatocyte lysosomal fragility in patients with hemochromatosis results in leakage of lysosomal enzymes and iron into the hepatocyte cytoplasm $(9,43)$. Indeed, our findings here confirm our previous observations that experimental iron overload also increases the fragility of hepatic lysosomes. It may appear unlikely that lysosomal enzymes released into the cytoplasm would damage cells directly since their enzymatic activity should be dramatically decreased at normal cytoplasmic pH. However, it is not known whether the cytosolic pH of iron-loaded hepatocytes is normal.
The mechanism of iron-induced lysosomal membrane fragility is thought to be via peroxidation of membrane lipids since incubation of isolated hepatocyte lysosomes with iron salts in vitro causes increased lysosomal membrane fragility and increased products of lipid peroxidation (44). However, evidence of in vivo lipid peroxidation of lysosomal membranes by iron overload has not been reported until now. Indeed, our results provide the first direct evidence that excess iron sequestered in hepatocyte lysosomes induces lipid peroxidation of lysosomal membrane lipids. This interpretation is supported by the very recent results of Houglum et al. (45) in an identical rat model of hepatic iron overload; using in situ immunohistochemistry, these workers demonstrated colocalization in hepatocytes of excess iron and aldehyde-protein adducts (i.e., products of lipid peroxidation) distributed in a vesicular pattern. Based on our results, it seems likely that this subcellular site of colocalization is the hepatocyte lysosome.

Although other interpretations are possible, we believe that the alterations we observed in lysosomal membrane fluidity are the result of iron-induced lipid peroxidation. Indeed, this interpretation is supported by in vitro experiments showing that lipid peroxidation decreases membrane fluidity in artificial liposomes (6). That the observed increase in anisotropy (i.e., decrease in fluidity) of lysosomal membranes after iron loading may be due to lipid peroxidation is suggested by our observation that malondialdehyde, an end product of lipid peroxidation, was increased in hepatic lysosomal membranes isolated from iron-loaded rats. We also examined other parameters known to alter membrane fluidity. No changes were noted in the ratios of cholesterol to phospholipid or lipid to protein, or in the double-bond index of lysosomal membranes. It is unclear at this time if the changes in fatty acid chain length or in fatty acid composition could account for the changes in fluidity or might rather represent an adaptive response to fluidity alterations.

Alterations in the fluidity of lysosomal membranes could have serious consequences for normal lysosomal function. For example, the fluidity of lysosomal membranes is likely important in lysosomal fusion with other intracellular vesicles and for the lysosomal uptake of macromolecules, including metalprotein complexes. Indeed, it is well known that membrane fluidity is an important parameter in regulating overall membrane function by affecting the orientation of integral membrane proteins, influencing membrane permeability, and modulating transmembrane transport processes (4). Perhaps this iron-induced alteration in lysosomal membrane fluidity with consequent altered fusion characteristics of lysosomes accounts for our previously reported observation that experimental iron overload is associated with a relative decrease of lysosomal enzyme output into bile (10); indeed, we had speculated previously that iron-induced suppression of fusion of hepatocyte lysosomes with the canalicular membrane accounted for the decreased biliary secretion of lysosomal enzymes we observed after iron loading.

Although our work establishes for the first time that experimental iron overload increases the pH of hepatocyte lysosomes, the exact mechanism of this effect is unclear (although likely related to peroxidation of lysosomal membrane lipids). Possible mechanisms include the inhibition by iron of the lysosomal proton ATPase either directly by similar mechanisms to free-radical injury of the $\mathrm{Na}^{+}-\mathrm{K}^{+}$ATPase pump $(46,47)$ or by depletion of cellular ATP (48); back diffusion of protons into 
the cytoplasm through a leaky lysosomal membrane; or alteration of membrane anion permeability affecting the transmembrane electrical potential (49). Regardless of the operative mechanism, the increase in lysosomal $\mathrm{pH}$ which we observed could adversely affect lysosomal digestion by impairing activities of lysosomal enzymes whose $\mathrm{pH}$ optima is generally below 5. Although additional studies are clearly necessary to distinguish among these various possible mechanisms and to determine the physiological consequences of increased intralysoso$\mathrm{mal} \mathrm{pH}$, our results demonstrate for the first time that increased intralysosomal iron directly affects at least one important lysosomal function.

Taken together, our data suggest the following model of hepatic injury by iron. Excess iron is sequestered within hepatocyte lysosomes by an as yet unclear mechanism; this excess iron initiates peroxidation of lysosomal membrane lipids. Lipid peroxidation causes decreased fluidity of lysosomal membranes and increased lysosomal fragility; it also induces alterations in lysosomal membrane lipid composition. These physicochemical alterations of the lysosomal membrane interfere with normal fusion of lysosomes with the canalicular membrane and with other organelles; the physicochemical alterations also inhibit the proper function of the lysosomal membrane proton pump, resulting in increased intralysosomal pH. As a result, the fusion-dependent digestive (i.e., heterophagy and autophagy) and excretory (i.e., exocytic discharge of lysosomal contents, including iron, into biliary canaliculi) activities of lysosomes is markedly impaired. If iron-induced lipid peroxidation is an important in vivo mechanism of lysosomal alterations, antioxidants such as vitamin $\mathrm{E}$ might be able to prevent or attenuate the lysosomal injury associated with iron overload. Additional experiments are necessary to test this model and to explore the relationship between these structural, physicochemical, and functional disturbances of hepatocyte lysosomes and the inevitable fibrosis and cirrhosis which results from a chronic excess in hepatic iron.

\section{Acknowledgments}

We would like to thank Shoji Ohkuma, Ph.D., and Thomas P. Moyer, $\mathrm{Ph} . \mathrm{D}$., for advice on biochemical, spectroscopic, and fluorometric techniques; Louis J. Kost, Pamela S. Tietz, James Tarara, Terri Toogood, and Peter J. Callahan for technical assistance; Susan Johnson for lipid analysis; Greg Gores for reviewing the manuscript; and Vel Woyczik and Shirley Hinze for typing the manuscript.

This work was supported by grants DK24031, DK34988, DK07198, and RR585 from the National Institutes of Health, by an American Gastroenterological Association Supplemental Grant, and by the Mayo Foundation, and the Hormel Foundation.

\section{References}

1. LaRusso, N. F. 1989. Hepatocyte lysosomes in intracellular digestion and biliary secretion. In Handbook of Physiology-the Gastrointestinal System III. Volume on Salivary, Gastric, Pancreatic, and Hepatobiliary Secretion, Chapter 33. J. G. Forte, editor. American Physiological Society, Bethesda, MD. 677-691.

2. Lucy, J. A. 1969. Lysosomal membranes. In Lysosomes in Biology and Pathology. Vol. 2. J. T. Dingle and H. B. Fell, editors. North-Holland Publishing Co., Amsterdam. 313-344.

3. Quinn, P. J. 1981. The fluidity of cell membranes and its regulation. Prog. Biophys. Mol. Biol. 38:1-104.

4. Schachter, D. 1984. Fluidity and function of hepatocyte plasma membranes. Hepatology (Baltimore). 4:140-151.

5. Stubbs, C. D., and A. D. Smith. 1984. The modification of mammalian membrane polyunsaturated fatty acid composition in relation to membrane fluidity and function. Biochim. Biophys. Acta. 779:89-137.
6. Bruch, R. C., and W. S. Thayer. 1983. Differential effect of lipid peroxidation on membrane fluidity as determined by electron spin resonance probes. Biochim. Biophys. Acta. 733:216-222.

7. Ohkuma, S., Y. Moriyama, and T. Takano. 1982. Identification and characterization of a proton pump on lysosomes by fluorescein isothiocyanate-dextran fluorescence. Proc. Natl. Acad. Sci. USA. 79:2758-2762.

8. Bassett, M. L., J. W. Halliday, and L. W. Powell. 1984. Genetic hemochromatosis. Semin. Liver Dis. 4:217-227.

9. Seymour, C. A., and T. J. Peters. 1978. Organelle pathology in primary and secondary haemochromatosis with special reference to lysosomal changes. $B r$. J. Haematol. 40:239-253.

10. LeSage, G. D., L. J. Kost, S. S. Barham, and N. F. LaRusso. 1986. Biliary excretion of iron from hepatocyte lysosomes in the rat: a major excretory pathway in experimental iron overload. J. Clin. Invest. 77:90-97.

11. Bacon, B. R., A. S. Tavill, G. M. Brittenham, C. H. Park, and R. O. Recknagel. 1983. Hepatic lipid peroxidation in vivo in rats with chronic iron overload. J. Clin. Invest. 71:429-439.

12. Wills, E. D. 1972. Effects of iron overload on lipid peroxide formation and oxidative demethylation by the liver endoplasmic reticulum. Biochem. Pharmacol. 21:239-247.

13. Kornbrust, D. J., and R. D. Mavis. 1980. Microsomal lipid peroxidation. 1. Characterization of the role of iron and NADPH. Mol. Pharmacol. 17:400407.

14. Bradford, M. 1976. A rapid and sensitive method for the quantitation of microgram quantities of protein utilizing the principle of protein dye binding. Anal. Biochem. 72:248-254.

15. LaRusso, N. F., and S. Fowler. 1979. Coordinate secretion of acid hydrolases in rat bile: hepatocyte exocytosis of lysosomal protein? J. Clin. Invest. 64:948-954.

16. deDuve, C., and R. Wattiaux. 1966. Functions of lysosomes. Annu. Rev. Physiol. 28:435-492.

17. Roeschlau, P., E. Bernt, and W. Gruber. 1974. Enzymatic determination of total cholesterol in serum. Z. Klin. Chem. Klin. Biochem. 12:226.

18. Takayama, M., S. Itoh, T. Nagasaki, and I. Tanimizu. 1977. A new enzymatic method for determination of serum choline-containing phospholipids. Clin. Chim. Acta. 79:93-98.

19. Holman, R. T., S. B. Johnson, J. M. Gerrard, S. M. Mauer, S. KapchoSandberg, and D. M. Brown. 1983. Arachidonic acid deficiency in streptozotocin-induced diabetes. Proc. Natl. Acad. Sci. USA. 80:2375-2379.

20. Holman, R. T., S. B. Johnson, and E. Kokmen. 1989. Deficiencies of polyunsaturated acids and replacement by non-essential fatty acids in plasma lipids in multiple sclerosis. Proc. Natl. Acad. Sci. USA. 86:4720-4724.

21. Smith, M. T., H. Thor, P. Hartzell, and S. Orrenius. 1982. The measurement of lipid peroxidation in isolated hepatocytes. Biochem. Pharmacol. 31:1926.

22. Spurr, A. R. 1969. A low viscosity epoxy embedding medium for electron microscopy. J. Ultrastruct. Res. 26:31-42.

23. Gomori, G. 1952. Microscopic Histochemistry, Principals and Practice. University of Chicago Press, Chicago.

24. Yamada, H., H. Hayashi, and Y. Natori. 1984. A simple procedure for the isolation of highly purified lysosomes from normal rat liver. J. Biochem. (Tokyo). 95:1155-1160.

25. Kapitulnik, J., M. Tshershedsky, and Y. Barenholz. 1979. Fluidity of the rat liver microsomal membrane: increase at birth. Science (Wash. DC). 206:843844.

26. Spencer, R. D., and G. Weber. 1969. Measurements of subnanosecond fluorescence lifetimes with a cross-circulation phase fluorometer. Ann. NY Acad. Sci. 158:361-376.

27. Ohkuma, S., and B. Poole. 1978. Fluorescence probe measurement of the intralysosomal $\mathrm{pH}$ in living cells and the perturbation of $\mathrm{pH}$ by various agents. Proc. Natl. Acad. Sci. USA. 75:3327-3331.

28. Berry, M. N., and D. S. Friend. 1969. High yield preparation of isolated hepatocytes. J. Cell Biol. 43:506-520.

29. Gores, G. J., L. J. Kost, L. J. Miller, and N. F. LaRusso. 1989. Processing of cholecystokinin by liver cells: evidence for peptide extraction via an organic anion transporter. Am. J. Physiol. 257:G242-G248.

30. Reijngoud, D. J., P. S. Oud, and J. M. Tager. 1976. Effects of ionophores on intralysosomal pH. Biochim. Biophys. Acta. 448:303-313.

31. LaRusso, N. F., L. J. Kost, J. A. Carter, and S. S. Barham. 1982. Triton WR-1339, a lysosomotropic compound, is excreted into bile and alters the biliary excretion of lysosomal enzymes and lipids. Hepatology (Baltimore). 2:209-215.

32. Dupourque, D., and F. Kern. 1969. Cytoplasmic and mitochondrial malate dehydrogenase from beef kidney. Methods Enzymol. 13:116-122.

33. Beaufay, H., A. A. Costesec, E. Feytmans, D. Thines-Sempoux, M. Robbi, and J. Berthet. 1974. Analytical study of microsomes and isolated subcellular membranes from rat liver. J. Cell Biol. 61:188-200.

34. Reeves, W. S., and G. M. Fimognari. 1966. Lactic dehydrogenase: heart (H4). Methods Enzymol. 9:288-294.

35. Bradford, W. D., J. G. Elchlepp, A. V. Arstila, B. R. Trump, and T. D. Kinney. 1969. Iron metabolism and cell membranes: relation between ferritin 
and hemosiderin in bile and biliary excretion of lysosomal contents. Am. J. Pathol. 56:201-228.

36. Park, C. H., B. R. Bacon, G. M. Brittenham, and A. S. Tavill. 1987. Pathology of dietary carbonyl iron overload in rats. Lab. Invest. 57:555-563.

37. Hultcrantz, R., B. Angelin, E. Bjorn-Rasmussen, S. Ewerth, and K. Einarsson. 1989. Biliary excretion of iron and ferritin in idiopathic hemochromatosis. Gastroenterology. 96:1539-1545.

38. Tribble, D. L., T. Y. Aw, and D. P. Jones. 1987. The pathophysiological significance of lipid peroxidation in oxidative cell injury. Hepatology (Baltimore). 7:377-387.

39. Halliwell, B., and J. M. C. Gutteridge. 1984. Oxygen toxicity, oxygen radicals, transition metals and disease. Biochem. J. 219:1-14.

40. Bacon, B. R., C. H. Park, G. M. Brittenham, R. O'Neill, and A. S. Tavill. 1985. Hepatic mitochondrial oxidative metabolism in rats with chronic dietary iron overload. Hepatology (Baltimore). 5:789-797.

41. Peters, T. J., and C. A. Seymour. 1976. Acid hydrolase activities and lysosomal integrity in liver biopsies from patients with iron overload. Clin. Sci. Mol. Med. 50:75-78.

42. Selden, C., M. Owen, J. M. P. Hopkins, and T. J. Peters. 1980. Studies on the concentration and intracellular localization of iron proteins in liver biopsy specimens from patients with iron overload with special reference to their role in lysosomal disruption. Br. J. Haematol. 44:593-603.

43. Peters, T. J., C. Selden, and C. A. Seymour. 1976. Lysosomal disruption in the pathogenesis of hepatic damage in primary and secondary haemochromatosis. Iron Metabolism, CIBA Foundation Symposium, Amsterdam. 51:317-329. 44. Mak, I. T., and W. B. Weglicki. 1985. Characterization of iron-mediated peroxidative injury in isolated hepatic lysosomes. J. Clin. Invest. 75:58-63.

45. Houglum, K., M. Filip, J. L. Witztum, and M. Chojkier. 1990. Malondialdehyde and 4-hydroxynonenal protein adducts in plasma and liver of rats with iron overload. J. Clin. Invest. 86:1991-1998.

46. Kim, M. S., and T. Akeca. 1987. $\mathrm{O}_{2}$ free radicals: cause of ischemia-reperfusion injury to cardiac $\mathrm{Na}^{+}-\mathrm{K}^{+}$-ATPase. Am. J. Physiol. 252:H252-H257.

47. Keeffe, E. B., B. F. Scharschmidt, N. M. Blankenship, and R. K. Ockner. 1979. Studies of relationships among bile flow, liver plasma membrane NaK-ATPase, and membrane microviscosity in the rat. J. Clin. Invest. 64:1590-1598.

48. Bacon, B. R., R. S. Britton, N. Dalton, and R. O'Neill. 1988. Effects of iron overload on hepatic nucleotide levels and energy state. Hepatology. 8:1240. (Abstr.)

49. Scharschmidt, B. F., and R. W. VanDyke. 1987. Proton transport by hepatocyte organelles and isolated membrane vesicles. Annu. Rev. Physiol. 49:69-85. 